\title{
Lipase-immobilized Cellulosic Capsules with Water Absorbency for Enhanced Pickering Interfacial Biocatalysis
}

Xiao He, ${ }^{a}$ Bernard P. Binks, ${ }^{b}$ Jinguang Hu, ${ }^{a}$ lan Gates and Qingye Lua,

a Department of Chemical and Petroleum Engineering, University of Calgary, 2500 University Drive, NW, Calgary, T2N 1N4, Alberta, Canada

${ }^{b}$ Department of Chemistry, University of Hull, Hull, HU6 7RX, UK

*Corresponding author: qingye.lu@ucalgary.ca

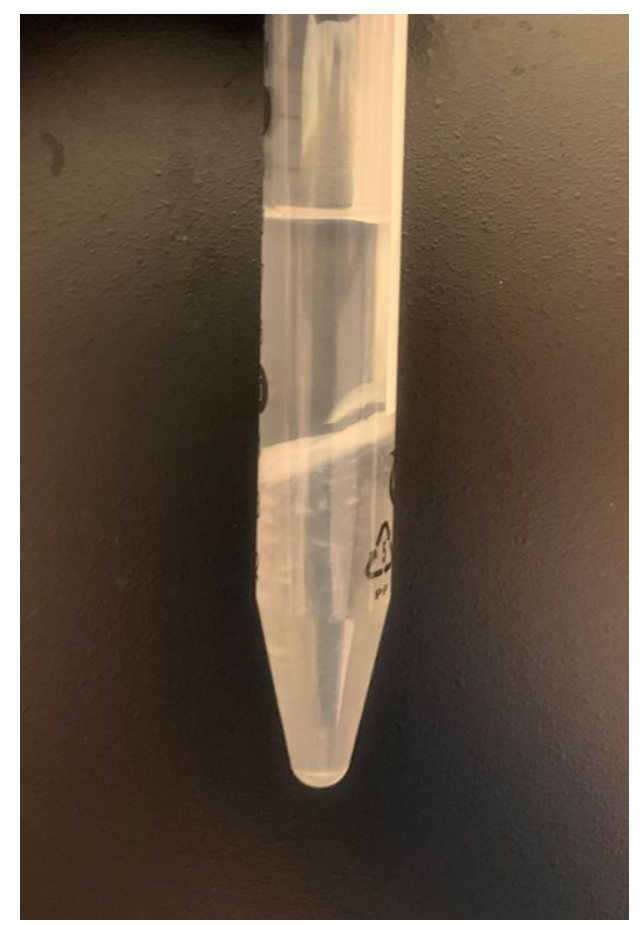

Figure S1. Phase-separated capsule-stabilized Pickering emulsion after centrifuging process. 

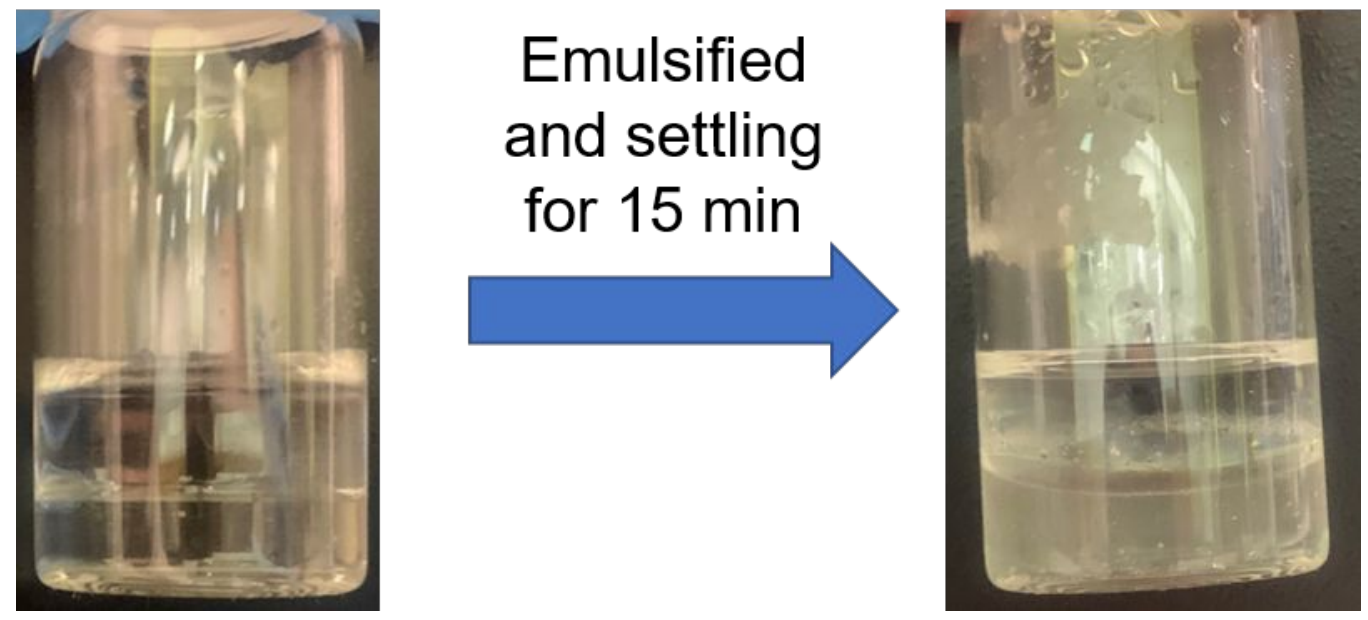

Figure S2. Emulsification of $4 \mathrm{~mL}$ toluene and $4 \mathrm{~mL} 1 \mathrm{wt} . \% \mathrm{CMC} /$ water solution, followed by settling for $15 \mathrm{~min}$, suggesting the interfacial inactivity of CMC on stabilizing emulsion.
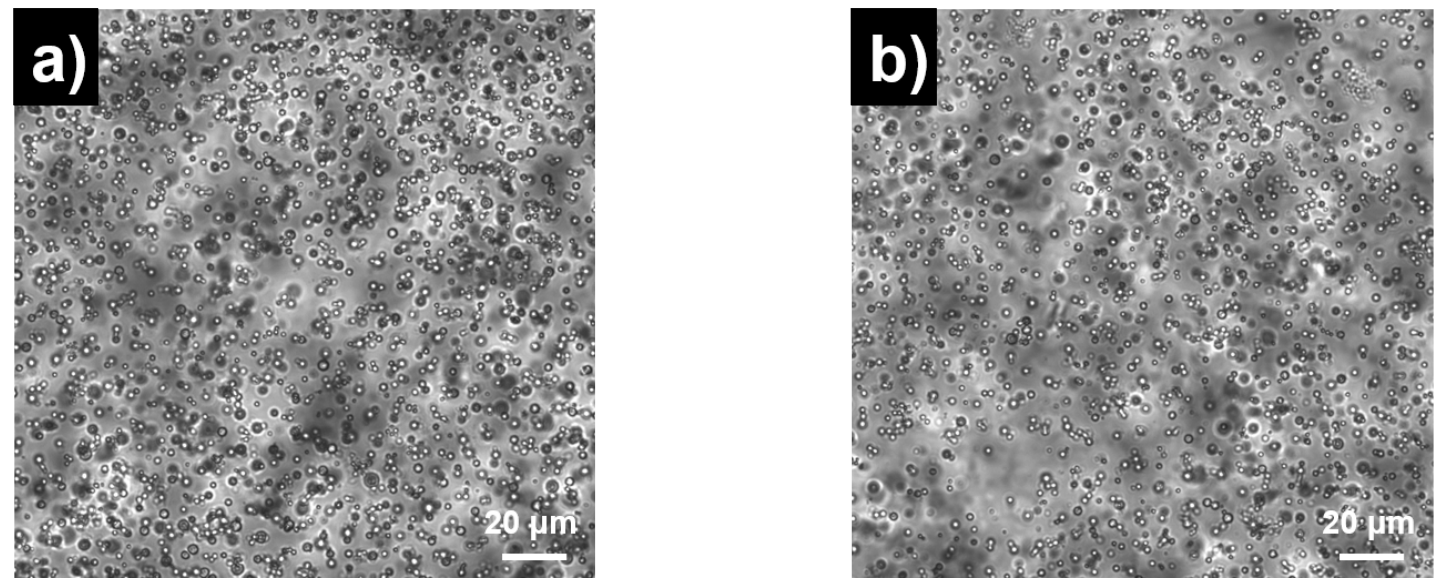

Figure S3. Microscope images of (a) water-in-1 wt.\% EC/toluene emulsion and (b) 1 wt.\% CMC/water-in-1 wt.\% EC/toluene emulsion. 


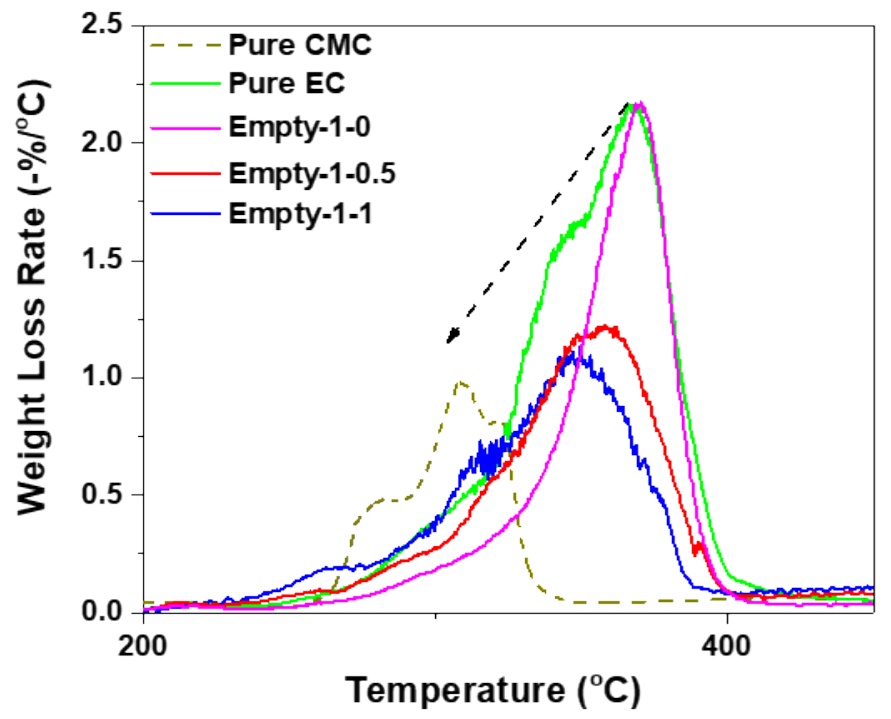

Figure S4. Weight loss rate of pure cellulosic materials and synthesized empty capsules. 

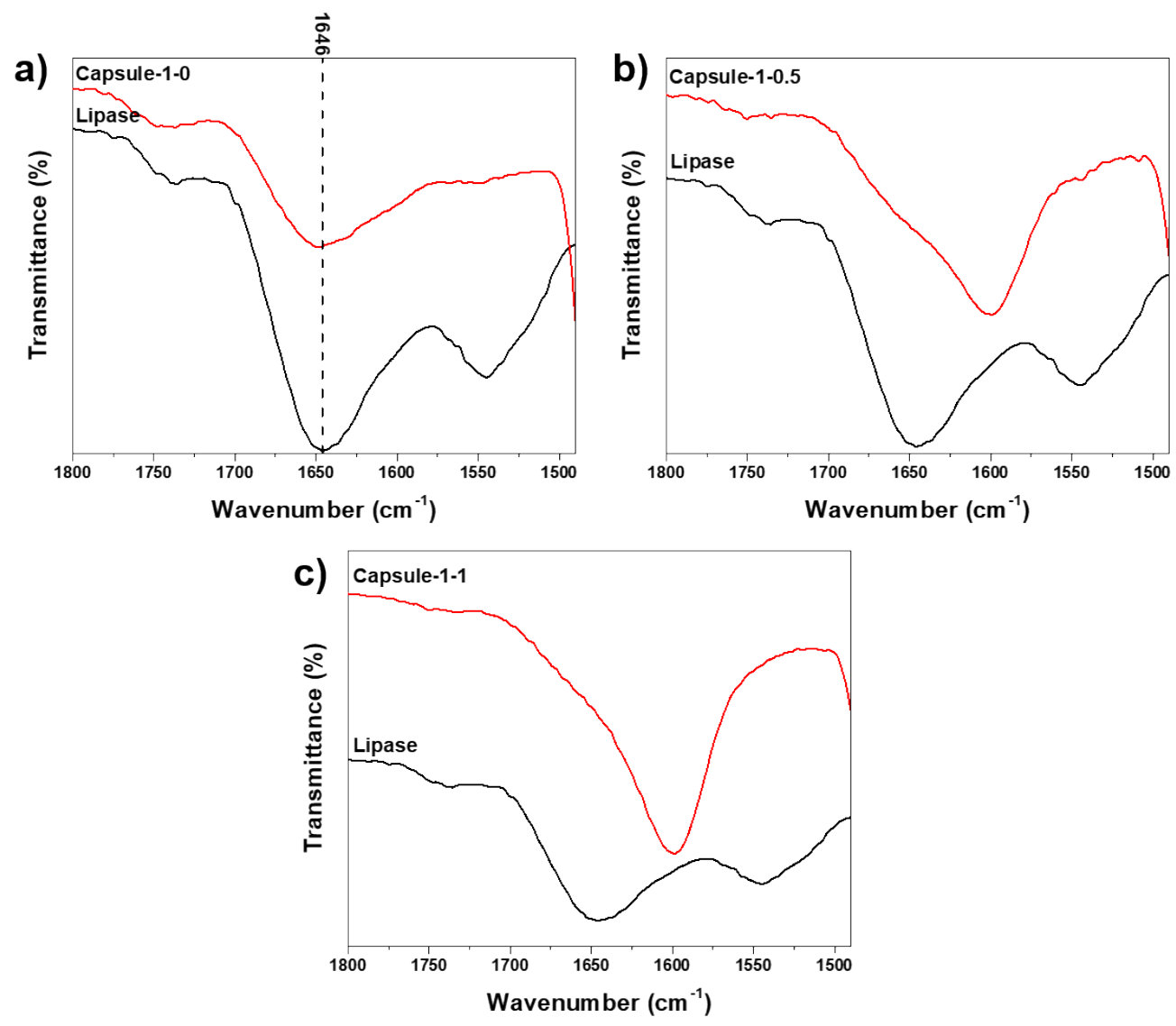

Figure S5. Narrow scan FTIR spectra of lipase and synthesized lipase-immobilized capsules.
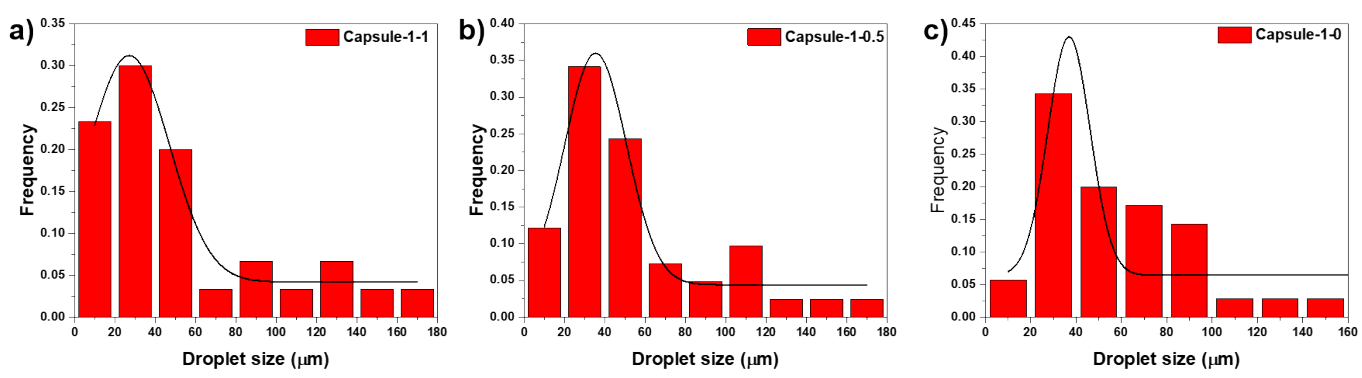

Figure S6. Size distributions of droplets in O/W Pickering emulsions stabilized by (a)

Capsule-1-0, (b) Capsule-1-0.5 or (c) Capsule-1-1 particles. 

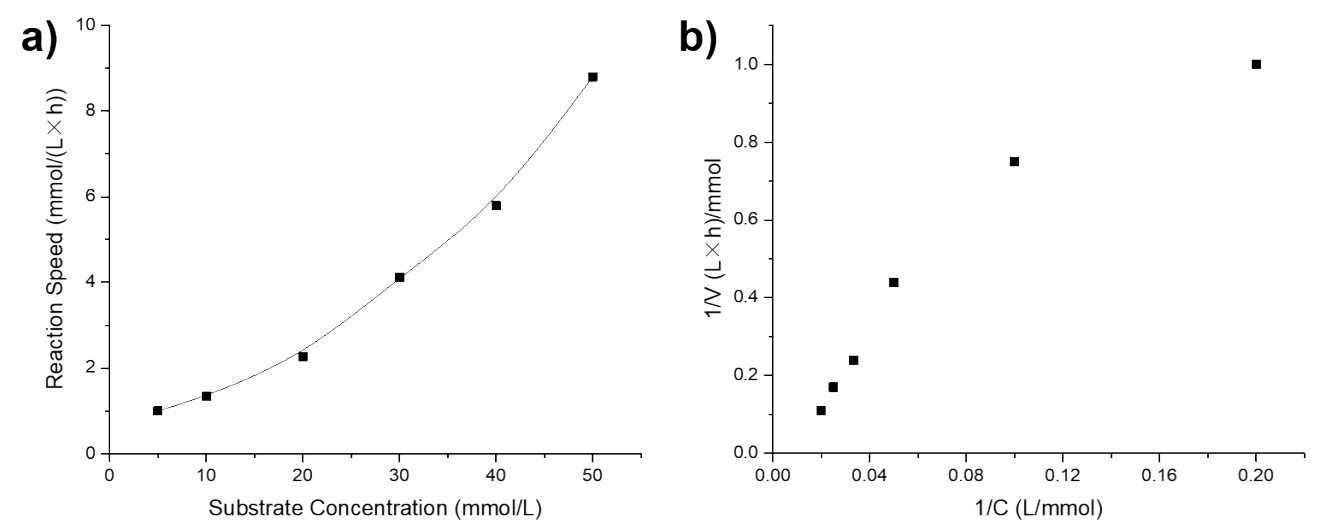

Figure S7. a) Reaction speed of Pickering interfacial catalysis system stabilized by Capsule-1-0.5 as a function of substrate (oleic acid + 1-octanol) concentration and b) corresponding Lineweaver-Burk double plot.
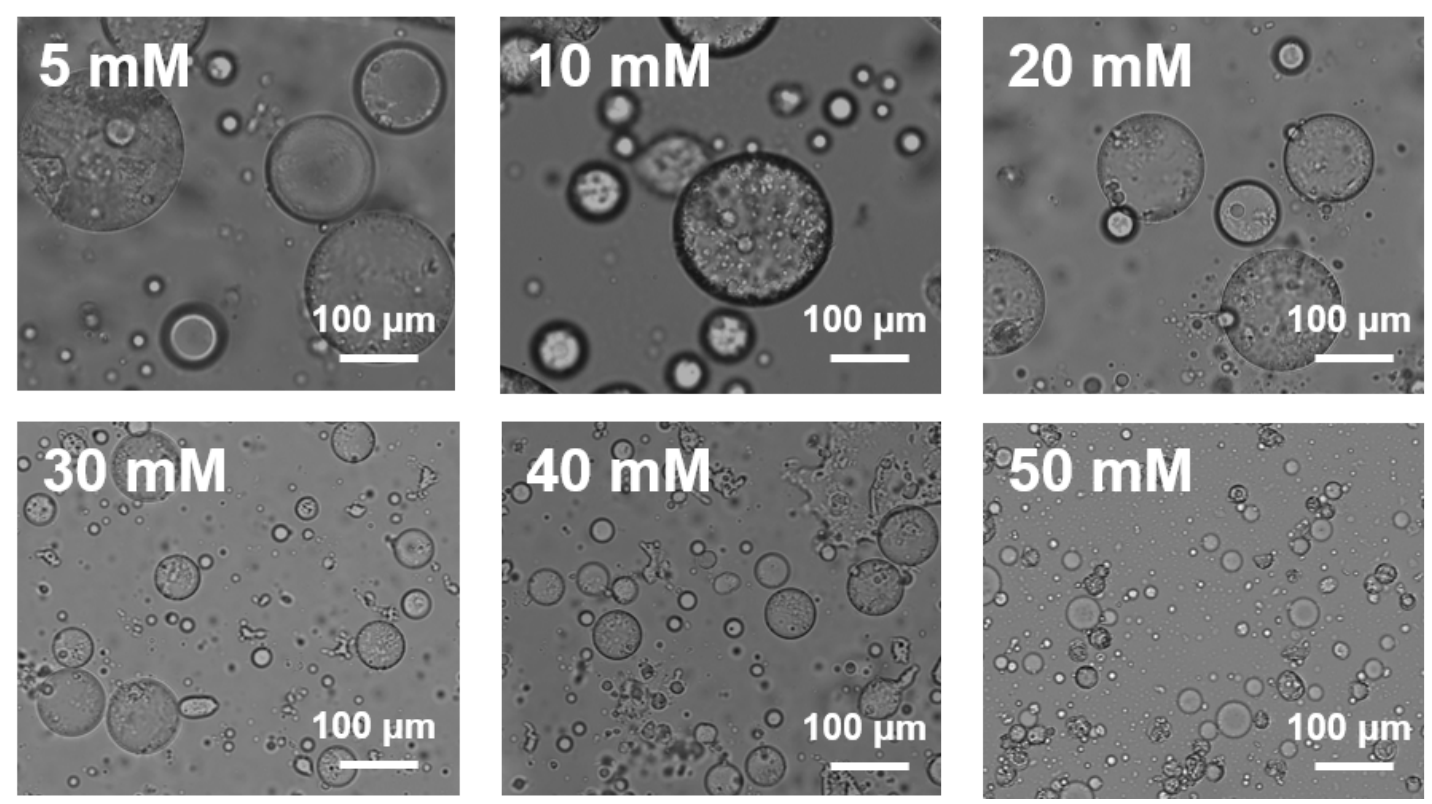

Figure S8. Hexane-in-water emulsion stabilized by Capsule-1-0.5 particles and different concentrations of oleic acid, demonstrating the smaller droplet size at higher concentration of oleic acid (the numbers in the figures represent the concentrations of oleic acid). 

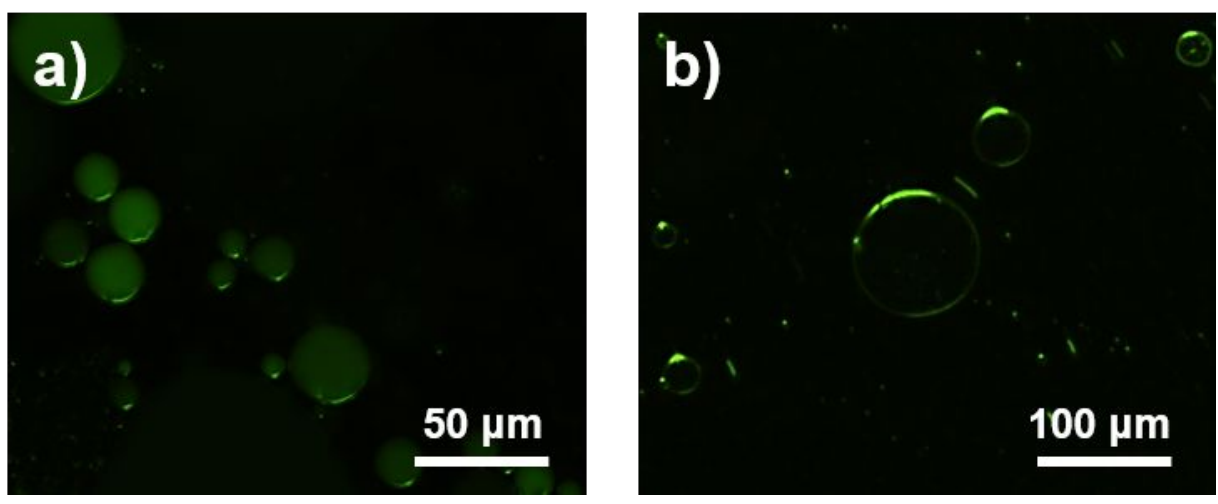

Figure S9. a) Water droplets as dispersed phase containing FITC-labeled lipases in 1 wt.\% EC/toluene and b) Pickering emulsion stabilized by the capsule-1-0 particles containing FITC-labeled lipase. 
Table S1. Catalytic performance of lipase-immobilized particles for catalysis in Pickering interfacial catalysis system.

\begin{tabular}{|c|c|c|c|c|c|}
\hline Enzyme & $\begin{array}{c}\text { Corresponding } \\
\text { Concentration } \\
\text { of Lipase } \\
(\mathrm{mg} / \mathrm{mL})\end{array}$ & Reactants & $\begin{array}{l}\text { Reaction } \\
\text { condition }\end{array}$ & $\begin{array}{c}\text { Final } \\
\text { Conversion }\end{array}$ & Reference \\
\hline $\begin{array}{l}\text { Lipase- } \\
\text { immobilized } \\
\text { alginate particle }\end{array}$ & 0.32 & $\begin{array}{c}\text { Hexanol } \\
+ \\
\text { hexanoic } \\
\text { acid }\end{array}$ & $\begin{array}{c}80 \mathrm{rpm}, \\
37^{\circ} \mathrm{C} \\
10 \mathrm{~h}\end{array}$ & $90 \%$ & 1 \\
\hline $\begin{array}{c}\text { Lipase- } \\
\text { immobilized } \\
\mathrm{Fe}_{3} \mathrm{O}_{4} @ \text { DhaTph }\end{array}$ & 13.28 & $\begin{array}{c}\text { Hexanol } \\
+ \\
\text { hexanoic } \\
\text { acid }\end{array}$ & $\begin{array}{c}55.5^{\circ} \mathrm{C} \\
1 \mathrm{~h}\end{array}$ & $95 \%$ & 2 \\
\hline $\begin{array}{l}\text { Lipase- } \\
\text { immobilized } \\
\text { mesoporous } \\
\text { silica sphere }\end{array}$ & 18 & $\begin{array}{c}\text { Lipoic } \\
\text { acid }+ \\
\text { sitosterol }\end{array}$ & $50^{\circ} \mathrm{C}, 6 \mathrm{~h}$ & $94.2 \%$ & 3 \\
\hline $\begin{array}{l}\text { Lipase- } \\
\text { immobilized } \\
\text { mesoporous } \\
\text { organicsilica } \\
\text { particle }\end{array}$ & N/A & $\begin{array}{l}\text { Ethanol } \\
+ \text { oleic } \\
\text { acid }\end{array}$ & $\begin{array}{c}200 \mathrm{rpm}, \\
35^{\circ} \mathrm{C} \\
24 \mathrm{~h}\end{array}$ & $64 \%$ & 4 \\
\hline $\begin{array}{c}\text { Lipase- } \\
\text { immobilized } \\
\text { ZIF } 8\end{array}$ & 0.528 & $\begin{array}{l}\text { 1-butanol } \\
\text { + vinyl } \\
\text { acetate }\end{array}$ & $\begin{array}{c}35^{\circ} \mathrm{C} \\
48 \mathrm{~h}\end{array}$ & $65 \%$ & 5 \\
\hline $\begin{array}{l}\text { Lipase- } \\
\text { immobilized } \\
\text { cellulosic } \\
\text { capsule }\end{array}$ & 0.125 & $\begin{array}{l}\text { Octanol } \\
+ \text { oleic } \\
\text { acid }\end{array}$ & $\begin{array}{c}25^{\circ} \mathrm{C} \\
48 \mathrm{~h}\end{array}$ & $76.3 \%$ & $\begin{array}{l}\text { This } \\
\text { work }\end{array}$ \\
\hline
\end{tabular}


Table S2. Dosages of materials used for preparing different lipase-immobilized capsules.

\begin{tabular}{cccc}
\hline Capsule & Oil phase & Aqueous Phase & Lipase (mg) \\
\hline Capsule-1-0 & $8 \mathrm{~mL} \mathrm{1} \mathrm{wt. \%}$ & $1.6 \mathrm{~mL}$ water & 4.7 \\
& EC/Toluene & & \\
Capsule-1-0.5 & $7.2 \mathrm{~mL} \mathrm{1} \mathrm{wt. \%}$ & $1.44 \mathrm{~mL} \mathrm{0.5} \mathrm{wt. \%}$ & 4.7 \\
& EC/Toluene & CMC/Water & \\
Capsule-1-1 & $6.53 \mathrm{~mL} 1 \mathrm{wt} \%$ & $1.31 \mathrm{~mL} \mathrm{1} \mathrm{wt. \%}$ & 4.7 \\
& EC/Toluene & CMC/Water & \\
\hline
\end{tabular}

\section{Reference:}

1. Yang, X.; Wang, Y.; Bai, R.; Ma, H.; Wang, W.; Sun, H.; Dong, Y.; Qu, F.; Tang, Q.; Guo, T. Pickering Emulsion-Enhanced Interfacial Biocatalysis: Tailored Alginate Microparticles Act As Particulate Emulsifier and Enzyme Carrier. Green Chem. 2019, 21, 2229-2233.

2. Yao, B. J.; Zhang, X. M.; Li, F.; Li, C.; Dong, Y. B. Fe $\mathrm{O}_{3} \mathrm{O}_{4} /$ Porphyrin Covalent Organic Framework Core-Shell Nanospheres as Interfacial Catalysts for Enzymatic Esterification. ACS Appl. Nano Mater. 2020.

3. Sun, T.; Dong, Z.; Wang, J.; Huang, F. H.; Zheng, M. M. Ultrasound-Assisted Interfacial Immobilization of Lipase on Hollow Mesoporous Silica Spheres in a Pickering Emulsion System: A Hyperactive and Sustainable Biocatalyst. ACS Sustain. Chem. Eng. 2020.

4. Jiang, Y.; Liu, X.; Chen, Y.; Zhou, L.; He, Y.; Ma, L.; Gao, J. Pickering Emulsion Stabilized by Lipase-Containing Periodic Mesoporous Organosilica Particles: A Robust Biocatalyst System for Biodiesel Production. Bioresour. Technol. 2014, 153, 278-283. 5. Lei, J.; Qi, L.; Luo, Z. Pickering Emulsion-Based Microreactors for Size-Selective Interfacial Enzymatic Catalysis. Front. Bioeng. Biotechnol. 2020, 8, 950. 